\title{
Interview with Hélène Gil by Hélène Guiral-Desnoës: How to rehabilitate lingual dysfunctions?
}

\author{
H. Guiral-Desnoës ${ }^{1}, \mathrm{H}$. Gil ${ }^{2}$ \\ 1 Qualified Specialist in Dento-Facial Orthopedics \\ 2 Independent Maxillofacial Physiotherapist in charge of teaching orofacial functional disorders \\ and pediatric orthodontics (Paris V)
}

H. Guiral-Desnoës: Are the techniques mentioned in the article written by $\mathrm{C}$. Duchateaux and Ms. Mangatalle-Louboutin all still topical?

H. Gil: The first step toward lingual rehabilitation is one of the main causes of failure in the treatment of lingual dysfunction. The major role of the rehabilitator is to make the patient aware that the success of treatment depends on them alone. What matters is not just giving a series of exercises to the patient-it is also convincing them to carry them out as often as possible so as to automatize the corrections. The success of the treatment is linked to the amount of time the patient invests in treatment. The persuasive strength of the rehabilitator is essential! In a difficult family environment, the physiotherapist will have to create a climate of confidence and develop a safe space so as to make the young patient want to attend the sessions.

The functions to be taken into account are the following:

- The resting position of the tongue: there should never be interdental contact. This is the most essential correction to obtain because it is the main source of dental dysmorphisms resulting from the high pressure caused by the lingual position at rest;

- Swallowing: atypical swallowing occurs during the phase of liquid collection (saliva, water) located at the anterior $1 / 3$ of the tongue, which is voluntarily controlled, and therefore is a possible target of rehabilitation. The swallowing of solids does not improve because it concerns the oesophageal part of the tongue whose movements are involuntary. To facilitate swallowing, it is necessary to improve the tone of the mylosternohyoid muscle and not the masseter muscles;

- Chewing: It is to be rehabilitated in the following cases: strict unilateral chewing, TMJ problems (malformation or condylar fracture), absence of occlusal meshing, or inverted occlusion;

- Phonation;

- Ventilation;

- Parafunctions (sucking on thumb, finger, teat, bottle, nail biting, tongue sucking,

Address for correspondence:

Hélène Guiral-Desnoës - 79, Avenue Pablo Picasso

92000 Nanterre

E-mail: helene.guiral@neuf.fr

Article Received: 21-11-2016. Accepted for publication: 11-11-2016.

This is an Open Access article distributed under the terms of the Creative Commons Attribution License (http://creativecommons.org/licenses/by/4.0), which permits unrestricted use, distribution, and reproduction in any medium, provided the original work is properly cited. 
strong tongue support in the palate and bruxism, biting tic of the lower lip). Bruxism in mixed dentition is a common occurrence, but should not be tolerated if it persists over time. Centered bruxism is the most binding for the muscles (pain) and eccentric bruxism is the most deleterious to the teeth (wear).

We must avoid grouping children in pairs; a half-hour session per patient and per physiotherapist is recommended. During the session, in addition to monitoring the exercise, the rehabilitator must devote time to motivate their patient. Outside of the sessions, the patient must practice his or her exercises daily for 15-20 min per day.

H. Guiral-Desnoës: Normalization of the Chateau triptych: rest position, swallowing, and phonation.

In your very informative article published in the DFO Journal in 2015 (49:277-292; Editorial Prize 2016 of the SPEPS Scientific Press), you also advocate rehabilitation using a device, can it be effective without accompanying neuromuscular education?

H. Gil: The Nocturnal Lingual Envelope (ELN) works in many cases but if the dyspraxia persists, it will be necessary for the patient to see a specialized rehabilitator.

H. Guiral-Desnoës: Should the ELN be accompanied by physiotherapy treatment?

H. Gil: If despite wearing the ELN and the patient's efforts, the lingual deposition persists, then it will be time to prescribe maxillofacial rehabilitation. Prescribing rehabilitation before or during orthodontic treatment would motivate the patient much more than after removal of the material. To begin without rehabilitation, will inevitably expose the patient to the risk of a re- lapse. To rehabilitate, before the fitting of the material or during the correction of the dysmorphism, will optimize the patient's compliance and makes the patient responsible for the success or failure of treatment. Therefore, lingual rehabilitation should be presented to the patient as "an active support" indispensable to the success of their orthodontic treatment.

H. Guiral-Desnoës: During the night, for example? Before? During? After care?

H. Gil: If when wearing the ELN, lingual malposition is necessarily corrected, it is essential to verify that when removing the device, the tongue remains in place. In the event of failure, the use of the ELN, as well as maxillofacial rehabilitation interrupted too early, raises the question of the quality of corrections.

H. Guiral-Desnoës: At what age should rehabilitation begin?

H. Gil: The child learns quickly but the adult is often more motivated! In summary, this is intimately linked to the motivation of each patient and the maturity of the children.

3-6 years: Between 3 and 6 years of age: we are talking about early cognitive development because the child will learn to feel, recognize, and try to correct bad postures and practices. However, as the child is often assisted by a parent to perform the exercises, the automation of corrections cannot be achieved.

$>6$ years (if maturity permits): The lingual correction is the same as in the adult, resting position of the tongue, swallowing, etc.

H. Guiral-Desnoës: Are fixed palatal devices (switch, Quad-Helix, Nance, transpalatine) an absolute contraindication to rehabilitation? Or can some 
of them be used as a reference point to position the tongue correctly (Tucat pearl on the anterior part of the Quad-Helix for example).

$H$. Gil: Rehabilitation is more difficult when there is something present in the mouth, especially on the palate, such as orthodontic appliances $(\mathrm{OH}$, palatal plates, plates, Nance tablets), the external perception of contact between tongue and palate is modified, and the attainment of the good tongue position is compromised.

H. Guiral-Desnoës: What do you think of the individualized anti-tongue grids attached to the mandible or maxilla, which are based on the lingual position of detrimental rest detected in the patient?

H. Gil: Stopping parafunctions by force is not a long-term solution because the patient may develop other parafunctions. The problem with tongue grids is that when the device is removed, the tongue will not necessarily stay in its proper position, because there are no mechanical constraints.

\section{Ventilation and obstructive sleep apnea (OSA)}

H. Guiral-Desnoës: Although it is obvious that ventilation disorders must be treated in parallel, is it possible to ask a mouth breather whose upper airways VAS are permanently obstructed to practice the exercises in to anticipate future healing, to take advantage at this favorable age, and to start establishing some habits? Or is it a lost cause?

$H$. Gil: It is essential to learn how to form good habits.

The treatment of OSA in adults is facilitated if rehabilitation is considered in advance.
Putting the tongue in place stimulates the desire to straighten up, the erectors also work on the posture. Failure to correct a posture defect can result in the tongue not being in the correct position. A mouth breather with lingual malposition often has this characteristic posture of the head thrown forward, rolled shoulders, lumbar hyperlordosis, hypotonic lips, and low tongue.

\section{Number of sessions required}

H. Guiral-Desnoës: Social security coverage is usually provided for 30 sessions, and we are often confronted with practitioners who, for reasons of organizing their appointments, follow the 30 sessions very regularly, once or twice per week, and then do not follow up afterward. Is this often enough? Is the number of sessions necessary dependent on the severity of the malfunction, or rather the involvement, or the age of the patient?

$H$. Gil: At least 20 sessions are required, after 10 sessions at the rate of one session per week, patients know all the exercises to practice. It is not because the patient does so willingly in front us that rehabilitation is complete. Additional sessions are required to verify the automation of new habits and postures, once every 2 weeks, then once a month, and then once every 2 months.

If all the issues detected in the initial visit have not been resolved, it is necessary to continue these sessions, and it can go on for a very long time in some cases.

The method used is Schultz's autogenic training method is the most effective way to eliminate bad habits and parafunctions. 


\section{Tongue is too short}

H. Guiral-Desnoës: What are the indications for a frenectomy?

H. Gil: A frenectomy is indicated in case of a bifurcated tongue (the tongue is in the shape of a heart when one sticks out the tongue) because in this case it is too long, and it is too difficult and painful to stretch it without great results. Surgery will save time on rehabilitation.

H. Guiral-Desnoës: What rehabilitation is used after the intervention?

$H$. Gil: It is imperative to see the patient on the same day, or at least the next day, to mobilize the tongue immediately. It is also good to see the patient a little before to reassure them and show them the exercises that will have to be done.

H. Guiral-Desnoës: To what extent can the well-practiced exercises stretch a shortened tongue?

$H$. Gil: A tongue can be stretched by $>2 \mathrm{~cm}$. Maintaining this result will be established by the automation of the tongue in the palate.

\section{Osteopathy}

H. Guiral-Desnoës: Are some osteopaths also able to perform procedures leading to the normalization of functions? If so, what are they?

H. Gil: Osteopathy will not directly correct the lingual dyspraxia but can contribute to the correction of it, resulting in a favorable muscular environment. In fact, tensions at the occipital crest or cervical spine, when they are not lifted, can slow down and prevent tongue retraction and the acquisition of good tongue position. Similarly, the correction of mouth breathing is difficult when these tensions persist.
H. Guiral-Desnoës: Finally, orthodontists are often confronted with parents who are in a hurry to use the device and who do not understand that the process of rehabilitation is just as necessary as the treatment to decrease the risk of recurrence.

Do you have any "tips" to make the patient and their parents aware of the need for such care?

H. Gil: Most parents feel that having their child undergo lingual rehabilitation is a financial cost and an additional time constraint. Yes, but it is the assurance of better support for orthodontic treatment, decreasing the time it takes to install the equipment, and preventing the teeth from moving again afterward. If the rehabilitation process was not followed, would the child need to continue wearing the device for many years? Will they have to wait until age 18 or undergo maxillofacial surgery, because the first orthodontic treatment was not successful?

Do their parents still want to pay for a new treatment, which will no longer be covered by social security? What guarantee can you give that it will hold this time? All of these questions can help parents and children realize that the success of the treatment also depends on their investment. I think it is important to make children and their parents aware that it is a real team effort. The architect cannot take the risk of building on unstable terrain. As physiotherapists, we will secure the ground; as orthodontists, you will build the house; and patients must commit themselves to do everything possible to maintain the structure.

Conflict of interest: The authors declare that there is no conflict of interest. 